\title{
A New Type of Single Carrier Conduction Rectifier on SiC
}

\author{
Satoshi Tanimoto ${ }^{1,3, a^{*}}$, Kenichi Ueoka ${ }^{1, b}$, Takaya Fujita ${ }^{1, c}$, Sawa Araki ${ }^{1, d}$, \\ Kazutoshi Kojima ${ }^{2, e}$,Toshiharu Makino ${ }^{2, f}$ and Satoshi Yamasaki ${ }^{2, g}$ \\ ${ }^{1}$ PEAL-DFAD, NISSAN ARC, Ltd., 1, Natsushima, Yokosuka 237-0061, Japan \\ ${ }^{2}$ ADPERC, Central 2, AIST, 1-1-1, Umezono, Tsukuba 305-8568, Japan \\ ${ }^{3} \mathrm{RCGI}$, Shibaura Institute of Technology, 3-7-5 Toyosu, Koto 135-8548, Japan \\ a*s-tanimoto@nissan-arc.co.jp, ${ }^{\mathrm{b}}$ ueoka@nissan-arc.co.jp, ct-fujita@nissan-arc.co.jp, \\ daraki@nissan-arc.co.jp, ${ }^{\mathrm{e}}$ kazu-kojima@aist.go.jp, ${ }^{\mathrm{f}}$ toshiharu-makino@aist.go.jp, \\ ${ }^{9}$ s-yamasaki@aist.go.jp
}

Keywords: Schottky, PiN, Rectifier, Diode, SiC, Unipolar action, Fast switching, Reverse recovery

\begin{abstract}
A new rectifier, called SPND or SNPD (Schottky-PN or -NP junction diode) and inherently showing low on-resistance and unipolar operation, was experimentally demonstrated for the first time on $4 \mathrm{H}-\mathrm{SiC}$. It is structured with an $\mathrm{n}^{-}$or a $\mathrm{p}^{-}$region of very low doping that is sandwiched and completely depleted between a Schottky junction and a one-sided PN junction. Either electrons or holes, but not both, contribute to the current conduction process. Clear and sharp rectifying properties are observed over the entire range of applied voltage.
\end{abstract}

\section{Introduction}

The Schottky barrier diode (SBD) shows very fast switching action based on its unipolar operation but suffers from high series resistance of the drift layer. A bipolar PiN diode can provide considerably lower on-resistance resulting from the storage effect of the minority carrier in the $i$ region; however, it causes an adverse reverse-recovery phenomenon in the turn-off transient and limits the switching frequency $[1,2]$. Some of the authors previously proposed a new type of rectifier, called the Schottky-PN junction diode (SPND or SNPD), which is potentially capable of resolving this ineludible dilemma, and substantiated its superior rectifying properties on a diamond semiconductor [3]. However, diamond is not yet a material of choice in the electronics industry at this time. The objective of this work is to demonstrate this rectifier on a mass-produced SiC substrate so as to encourage its broad use.

\section{Structure and Conduction Mechanism}

The new diode is structured with an $\mathrm{n}^{-}$or a $\mathrm{p}^{-}$region of very low doping that is sandwiched and completely depleted between a Schottky junction and a one-sided PN junction. The forward and

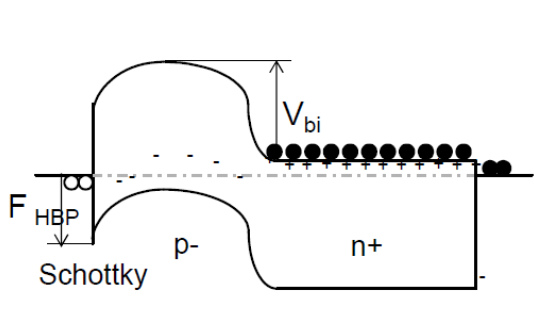

(a)

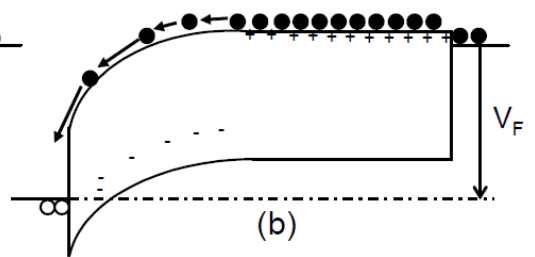

(b)

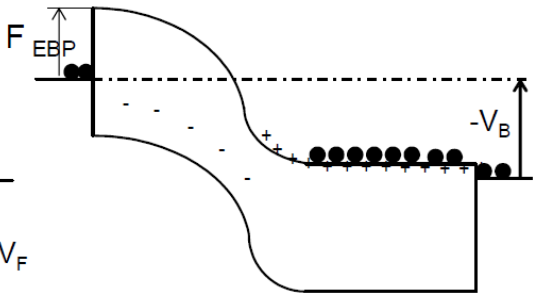

(c)

Fig. 1 Energy band diagrams for a Schottky-PN junction diode (SPND): (a) zero bias state, (b) forward bias state, and (c) reverse bias state. 


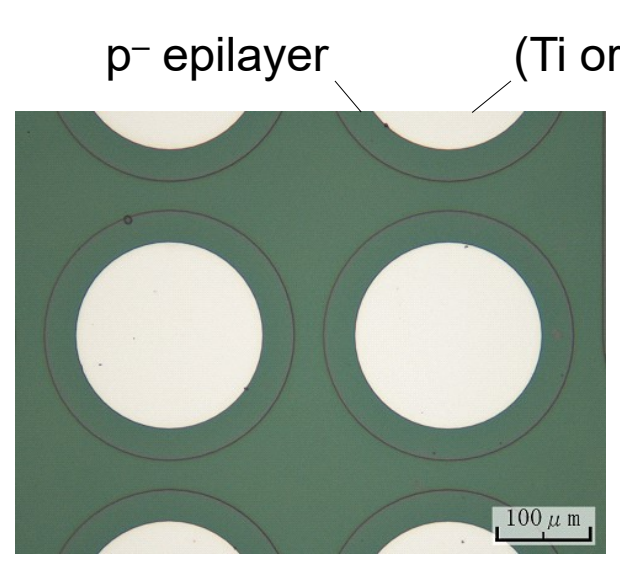

(a)

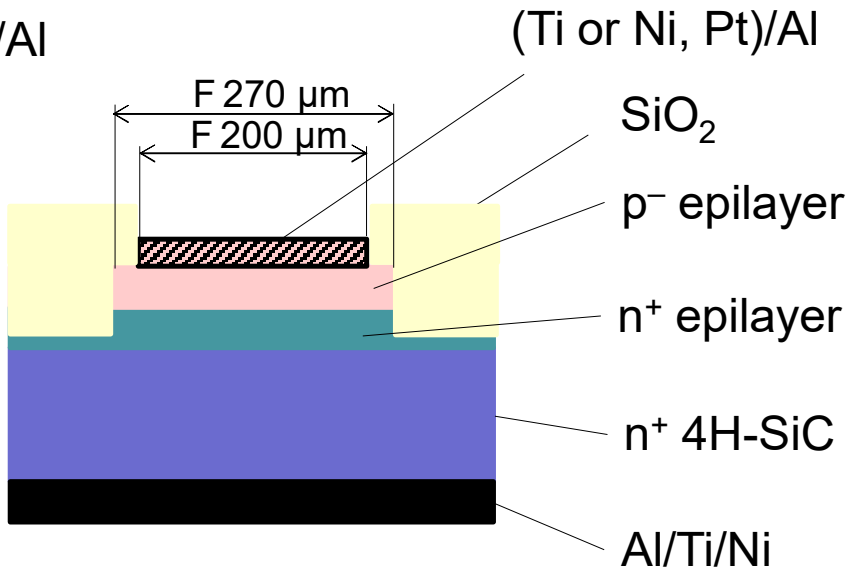

(b)

Fig. 2 Structure: (a) array of SPNDs (plan view photograph) and (b) cross-section of a single SPND.

reverse directions of the current flow correspond to those of an embedded PN junction. Figure 1 shows energy band diagrams for the SPND, where the low doping region is made of the p-type. When forward biased (Fig. 1b), the built-in potential $V_{b i}$ is reduced and free electrons are injected from the $\mathrm{n}^{+}$region into the $\mathrm{p}^{-}$region, thus allowing significant forward current to flow. All of the injected electrons can diffuse and drift to the Schottky junction without recombining with holes because the $\mathrm{p}^{-}$ region is completely depleted. As a result, the $\mathrm{p}^{-}$region is expected to show very low resistance in the forward conduction state [3]. It should also be emphasized that none of the holes contribute to the current conduction process. This signifies that the SPND inherently shows unipolar operation [3]. When the diode is reversely biased (Fig. 1c), most of the blocking voltage is shared throughout the pregion.

\section{Device Preparation}

An array of SPNDs with the structure and dimensions as displayed in Fig. 2 was fabricated on some (0001) Si face $8^{\circ}$ off-cut n-type low resistivity $4 \mathrm{H}-\mathrm{SiC}$ substrates $\left(\mathrm{N}_{\mathrm{D}}=7 \times 10^{18} \mathrm{~cm}^{-3}\right)$ using a standard semiconductor device production facility. Fabrication started with successive epitaxial growth of an $\mathrm{n}^{+}$buffer layer $\left(\mathrm{t}=0.83 \mu \mathrm{m}\right.$ thick, nitrogen $\left.\mathrm{N}_{\mathrm{D}}=1.5-1.7 \times 10^{18} \mathrm{~cm}^{-3}\right)$ and a $\mathrm{p}^{-}$layer $(\mathrm{t}=0.16 \mu \mathrm{m}$ thick, aluminum $\mathrm{N}_{\mathrm{A}}<10^{16} \mathrm{~cm}^{-3}$ ) on the bulk SiC substrate. Subsequently, after mesa-etching the $\mathrm{p}^{-}$epilayer, the substrate surface was covered with a $1-\mu \mathrm{m}$-thick field oxide $\left(\mathrm{SiO}_{2}\right)$ formed by thermal oxidation and thermal CVD. Contact windows were then opened in the field oxide located over the mesa surface, and thin $\mathrm{Ti}, \mathrm{Ni}$ or Pt as Schottky barrier metals and thick Al as an overlay metal were deposited selectively therein in the same manner as explained in [4]. The Schottky electrode area was $\mathrm{S}=$ $3.14 \times 10^{-4} \mathrm{~cm}^{2}(200 \mu \mathrm{m}$ in diameter). Finally, non-alloyed $\mathrm{Al}$ ohmic contacts with $\mathrm{Ni} / \mathrm{Ag}$ overlays were formed on the back side of the $\mathrm{n}^{+}$substrate by electron beam evaporation [5]. The thickness and $\mathrm{N}_{\mathrm{D}}$ of the $\mathrm{n}^{+}$buffer layer and the thickness of the $\mathrm{p}^{-}$layer were determined by using a SIMS (secondary ion mass spectroscopy) depth profiling technique.

\section{Results and Discussion}

Figure 3 shows the static current-voltage (I-V) characteristics for a single SPND fabricated with different Schottky barrier metals. As can be easily seen, clear and sharp rectifying properties are observed over the entire range of applied voltage, indicating that the Schottky-PN diode can function properly as a rectifier on $\mathrm{SiC}$, just as on diamond in [3]. The current density was $318 \mathrm{~A} / \mathrm{cm}^{2}$ at the maximum current flow $(100 \mathrm{~mA})$ in the forward conduction state. The differential specific

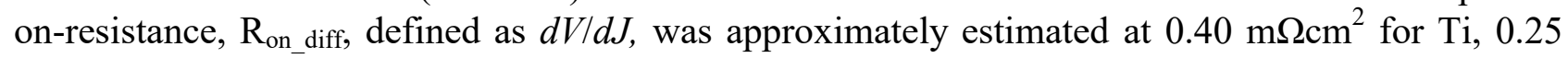


$\mathrm{m} \Omega \mathrm{cm}^{2}$ for $\mathrm{Ni}$ and $0.37 \mathrm{~m} \Omega \mathrm{cm}^{2}$ for Pt at $100 \mathrm{~mA}$. Note that these $R_{\text {on_diff values are for reference }}$ only because our SPNDs had a very small area. The knee voltage, $\mathrm{V}_{\text {knee, }}$ was approximately estimated at $0.22 \mathrm{~V}$ for $\mathrm{Ti}, 1.10 \mathrm{~V}$ for $\mathrm{Ni}$ and 1.48 $\mathrm{V}$ for Pt. It is interesting to note that $\mathrm{V}_{\text {knee }}$ significantly depends on the hole barrier height, $\Phi_{\mathrm{HBP}}$, of the Schottky junction to the $\mathrm{p}$ region. This is because the larger $\Phi_{\mathrm{HBP}}$ pulls down $\mathrm{V}_{\mathrm{bi}}$ more strongly (Fig. 1b). The Ti-SPND had a large leakage current in the reverse bias state. This can be interpreted as follows. Ti usually shows a very low electron barrier height, $\Phi_{\mathrm{EBP}}$, on well cleaned $\mathrm{SiC}$, as reported previously in [5]. In this study, conduction electrons in $\mathrm{Ti}$ were easily injected into the conduction band of the $\mathrm{p}^{-}$layer by applying a low reverse bias, which resulted in the flow of the large leakage current as seen in Fig. 3.

The capacitance-voltage $(C-V)$ characteristics

for each single SPND with Schottky barrier metals of Ni and Pt were measured at $1 \mathrm{kHz}$, but those of Ti-SPND could not be measured because of the large leakage current. Figure 4 shows (a) $1 / C^{2}$ and (b) the depletion layer width $\left(W_{\mathrm{S}}\right)$ as a function of $V$, where the data in the voltage range definitely over $V$ $=\mathrm{V}_{\text {knee }}$ in Fig. 3 are invalid since the conduction current made it difficult to measure $C$ properly. $W_{\mathrm{S}}$ was converted from $C$ by using the relationship of $W_{\mathrm{S}}=\epsilon_{0} \cdot \epsilon_{\mathrm{SiC}} \cdot \mathrm{S} / \mathrm{C}$, where $\epsilon_{0}\left(=8.85 \times 10^{14} \mathrm{~F} / \mathrm{cm}\right)$ and $\epsilon_{\mathrm{SiC}}\left(=9.8 \epsilon_{0}\right)$ are permittivity in a vacuum and the dielectric constant of $\mathrm{SiC}$, respectively. Figure $4 \mathrm{~b}$ indicates that $W_{\mathrm{S}}$ at $V=\mathrm{V}_{\text {knee }}$ or slightly above was consistent with the physical thickness of the $\mathrm{p}^{-}$ region, i.e. $\mathrm{t}=0.16 \mu \mathrm{m}$. This finding is evidence validating the energy band diagram in Fig. $1 \mathrm{~b}$. A linear increase in $1 / C^{2}$ (slope $l_{1}$ and $l_{2}$ ) observed in the reverse bias region in Fig. 4a represents a gradual extension of the depletion layer width within the $\mathrm{n}^{+}$buffer layer with a decline of $V$. Since the slopes $l_{1}$ and $l_{2}$ correspond to a value of $2 /\left(\epsilon_{0} \cdot \epsilon_{\mathrm{SiC}} \cdot \mathrm{q} \cdot \mathrm{S}^{2} \cdot \mathrm{N}_{\mathrm{D}}\right)$, where $\mathrm{q}$ is an elementary charge [1, 2], we obtained the $\mathrm{N}_{\mathrm{D}}$ (precisely, $\mathrm{N}_{\mathrm{D}}-\mathrm{N}_{\mathrm{A}}$ ) values of the $\mathrm{n}^{+}$buffer layer in Ni- and Pt-SPNDs, that is, $\mathrm{N}_{\mathrm{D}}=$ $1.8 \times 10^{18} \mathrm{~cm}^{-3}$ for Ni-SPND and $\mathrm{N}_{\mathrm{D}}=1.7 \times 10^{18} \mathrm{~cm}^{-3}$ for Pt-SPND. These values were nearly identical to those attained by the SIMS analysis.

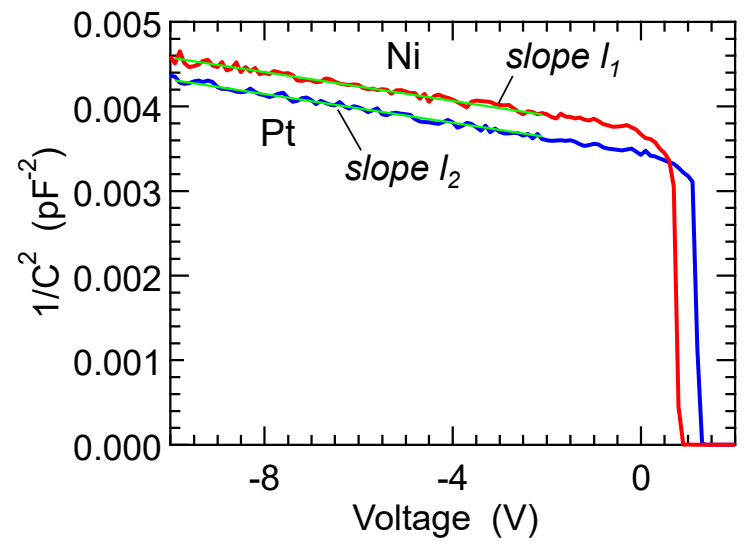

(a)

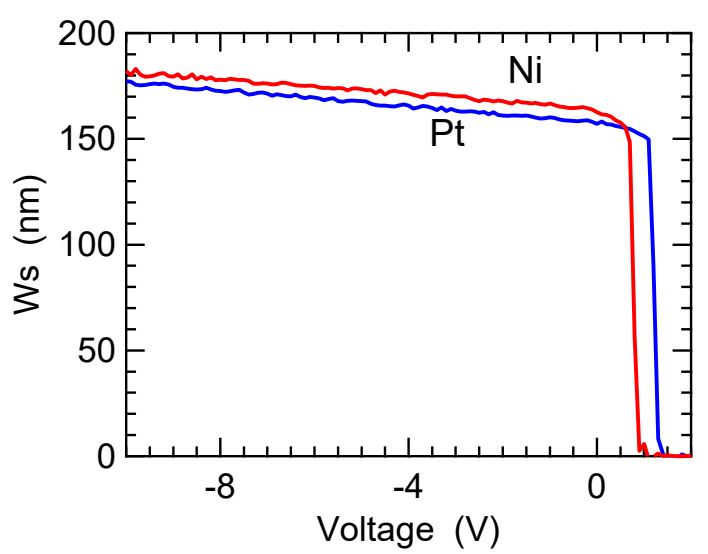

(b)

Fig. 4 Capacitance-voltage $(C-V)$ characteristics of a single SPND with different Schottky barrier metals of $\mathrm{Ni}$ and $\mathrm{Pt}$ : (a) $1 / C^{2}$ and (b) depletion region width $\mathrm{W}_{\mathrm{S}}$ as a function of applied voltage. 
Finally, the average dielectric breakdown field of the SPND in the blocking state is discussed. The breakdown voltages experimentally obtained were $V_{B}=-54.0 \mathrm{~V}$ for Ni-SPND and $V_{B}=-59.5 \mathrm{~V}$ for Pt-SPND (Fig. 3). Assuming that the linear relationship between $1 / C^{2}$ and $\mathrm{V}$ observed in the reverse bias region in Fig. 4a holds true until $\mathrm{V}_{\mathrm{B}}$, we obtained $C$ and $W_{\mathrm{S}}$ at $\mathrm{V}_{\mathrm{B}}$. Thus, the average breakdown fields were estimated to be $\mathrm{E}_{\mathrm{B}}\left(=\mathrm{V}_{\mathrm{B}} / W_{\mathrm{S}}\right)=2.2 \mathrm{MV} / \mathrm{cm}$ for Ni-SPND and $2.3 \mathrm{MV} / \mathrm{cm}$ for Pt-SPND. These $\mathrm{E}_{\mathrm{B}} \mathrm{S}$ approximately correspond to $80 \%$ of the dielectric breakdown field $(2.8 \mathrm{MV} / \mathrm{cm})$ reported previously [6].

\section{Summary}

A new rectifier, called SPND or SNPD and inherently showing low on-resistance and unipolar operation, was experimentally demonstrated for the first time on $4 \mathrm{H}-\mathrm{SiC}$. It is structured with an $\mathrm{n}^{-}$or a $\mathrm{p}^{-}$region of very low doping that is sandwiched and completely depleted between a Schottky junction and a one-sided PN junction. Either electrons or holes, but not both, contribute to the current conduction process. Clear and sharp rectifying properties were observed over the entire range of applied voltage. The SPND on $4 \mathrm{H}-\mathrm{SiC}$ conducted a current density of at least more than $300 \mathrm{~A} / \mathrm{cm}^{2}$. Average breakdown fields in the depletion layer were estimated to be $2.3 \mathrm{MV} / \mathrm{cm}$, corresponding to approximately $82 \%$ of the dielectric breakdown field $(2.8 \mathrm{MV} / \mathrm{cm})$ widely known for $4 \mathrm{H}-\mathrm{SiC}$.

\section{Acknowledgment}

This work was partly conducted under the Potentiality Verification Stage Program of the Japan Science and Technology Agency (JST).

\section{References}

[1] J. Baliga, Chapters 4 and 5, Fundamentals of Power Semiconductor Devices, Springer, New York, 2008.

[2] C. C. Hu, Chapter 4, Modern Semiconductor Devices for Integrated Circuits, Prentice Hall, 2010.

[3] T. Makino, S. Tanimoto, Y. Hayashi, H. Kato, N. Tokuda, M. Ogura, D. Takeuchi, K. Oyama, H. Ohashi, H. Okushi, and S. Yamasaki, Diamond Schottky-pn diode with high forward current density and fast switching operation, Appl. Phy. Lett., 94 (2009) 262101.

[4] S. Tanimoto, H. Okushi, and K. Arai, Ohmic contacts for power devices on SiC, in: W.J. Choyke, H. Matsunami and G. Pensl (Eds.), Silicon Carbide-Recent Major Advances, Springer: Berlin, 2003, pp. 652-669.

[5] S. Tanimoto, M. Inada, N. Kiritani, M. Hoshi, H. Okushi, and K. Arai, Single contact-material MESFETs on 4H-SiC, Mater. Sci. Forum, 457-460 (2004) 1221-1224.

[6] O. Madelung (Ed.), Data in Science and Technology, Semiconductors, Group IV Element and III-V Compound, Springer-Verlag, Berlin, 1991. 